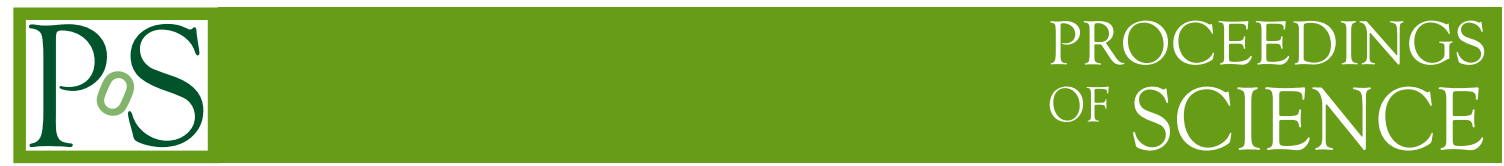

\title{
Observable signatures of lifecycle of PAHs in HII complexes
}

\author{
Maria Khramtsova*, Dmitry Wiebe \\ Institute of Astronomy of Russian Academy of Sciences \\ E-mail: khramtsova@inasan.ru
}

\begin{abstract}
We study the ratio between two mid-infrared emission bands, $8 \mu \mathrm{m}$ and $24 \mu \mathrm{m}$, in extragalactic HII complexes. Our sample includes more than a hundred complexes for which infrared (Spitzer), ultraviolet (GALEX) and optical spectroscopic data are available. We analyze the correlation of the $F_{8} / F_{24}$ ratio with metallicity, hardness of ionizing radiation field and age of an HII complex. We suggest that evolutionary effects can be seen for the $F_{8} / F_{24}$ ratio, but these effects are different at different metallicities.
\end{abstract}

The Life Cycle of Dust in the Universe: Observations, Theory, and Laboratory Experiments 18-22 November, 2013

Taipei, Taiwan

\footnotetext{
* Speaker.
} 


\section{Introduction}

Polycyclic aromatic hydrocarbons (PAHs) are ubiquitous in the Universe. Because of their small sizes (of the order of 10-100 $\AA$ ) they can be heated up to high temperatures even by a single photon, in other words, the heating of PAHs is stochastic. This feature makes their heating most efficient in objects with a strong UV radiation field, so HII regions/complexes are the objects where PAH emission is very bright. PAH emission is seen in nearly all HII regions both in our Galaxy and in external galaxies, so these objects are suitable to study PAH properties and their life cycle.

Most extragalactic studies are based on observations of entire galaxies, because the resolution of available instruments (e.g. Spitzer) is still not sufficient to study individual HII regions. However, there are some works devoted to HII complexes in Magellanic Clouds or in other nearby galaxies [3]. One of the most interesting features of PAHs is their correlation with metallicity: low metallicity objects have low abundance of PAHs while the abundance increases with growing metallicity both on a global scale (e.g. [1]) and in individual HII complexes [3]. There is no unique interpretation of this, though possible scenarios including efficient destruction by a harsher radiation field [5] or less efficient formation [2] in low metallicity environments were suggested.

In this work we study possible correlations between PAH emission, hardness of radiation field and age of HII complexes. Firstly, we try to elucidate what is a crucial factor that controls abundance of PAHs in HII complexes. Secondly, we check whether there are evolutionary changes in PAH emission.

\section{Data}

Our sample includes about a hundred HII complexes in nine nearby galaxies that have different morphological types and metallicities. Some of them are included in SINGS and KINGFISH surveys. Complexes in the studied galaxies are distributed nearly evenly by metallicity in a range from 7.5 to 8.8 (in units of $12+\log (\mathrm{O} / \mathrm{H})$ ). This allows us to study possible correlations at different chemical conditions.

We perform aperture photometry for HII complexes using Spitzer data at wavelengths of 3.6, 4.5, 5.8 and 8.0 and $24 \mu \mathrm{m}$ and data at near and far ultraviolet bands (FUV, NUV). Also we use spectroscopic measurements from the literature and using our own data taken with the 6-m telescope of Special Astrophysical Observatory of Russian Academy of Sciences.

\section{Physical parameters of HII complexes}

Throughout this work the following parameters briefly described below are considered.

Ratio $\mathbf{F}_{\mathbf{8}} / \mathbf{F}_{\mathbf{2 4}}$. To estimate PAH abundance in HII complexes we use results of aperture photometry from Spitzer, namely a ratio of fluxes in the $8 \mu \mathrm{m}$ and $24 \mu \mathrm{m}$ bands. It is conventionally adopted that the $8 \mu \mathrm{m}$ emission arises from PAHs, while the $24 \mu \mathrm{m}$ emission comes from very small grains (VSGs). Though the ratio of these fluxes is not a straightforward index of PAH abundance it can be used as an indirect indicator of a mass fraction of PAHs in HII complexes or galaxies as it was shown in the works of [3]. 
Metallicity. Metallicity of HII complexes is estimated mostly by the 'ONS' method based on the brightest lines of oxygen, nitrogen and sulfur or the 'NS'-method in the case of a lack of oxygen line measurements.

Ages of HII complexes. We estimate the age of the HII complexes using an equivalent width of $\mathrm{H}_{\beta}$ line. In [4] some expressions are given to determine the starburst age for different metallicities $\left(Z / Z_{0}=0.05,0.2,0.4,1.0,2.0\right)$. These expressions are adopted in this work to estimate ages of HII complexes.

Radiation field. Hardness of interstellar radiation field in HII complexes is determined in two ways. Firstly, FUV-NUV is used as an indicator of the UV field hardness. Secondly, the ratio $I([O I I I] \lambda 5007) / I\left(H_{\beta}\right)$ is used as a quantity that is sensitive to the ionizing UV field.

\section{Results and discussion}

We demonstrate a well-known correlation between PAH emission and metallicity in Fig. 1 (a). The correlations between the $F_{8} / F_{24}$ ratio and indicators of radiation field hardness are presented in Fig. 1 (b, c). Actually, our indicators of radiation hardness are related to metallicity, so the correlations in Fig. 1 (b, c) are expected. Low metallicity environments have a harsher radiation field than high metallicity ones, wherein PAH fraction decreases as well. So the hardness can be a factor that influences the PAH abundance. Arguably, PAHs can be destroyed more efficiently in harder radiation fields.

The correlation between the $F_{8} / F_{24}$ ratio and metallicity shows considerable variation in various environments. This could mean that PAH emission is controlled also by other factors, not only by chemical conditions. In this work we probe whether there is any evolutionary changes for the $F_{8} / F_{24}$ ratio. To consider this we divide all the complexes into 5 groups by metallicity to have approximately equal number of complexes in each group. We demonstrate how the $F_{8} / F_{24}$ ratio changes with age of HII complex in each group in Fig. 2. To estimate the goodness of a correlation the Spearman rank correlation coefficient is used. The $F_{8} / F_{24}$ ratio increases with age at low metallicity, while at higher metallicity the correlation disappears and at the highest metallicity it turns to the inverse one, that is, $F_{8} / F_{24}$ decreases with age. Firstly, such correlations imply that $F_{8} / F_{24}$ may increase or decrease inside an HII complex during its evolution. Secondly, we may suggest that abundances of both PAHs and VSGs may change with time. Probably, a balance between destruction and formation processes of PAHs and VSGs plays a role here. In particular, if destruction of VSGs leads to formation of PAHs, then this process may have different efficiency at different metallicity. For example, the following explanation is possible. At low metallicity, the rate of PAH destruction can be partially compensated by their formation due to VSG destruction, i.e. PAH destruction is less efficient than VSG destruction. So we see a growth of the $F_{8} / F_{24}$ ratio with age. With increasing metallicity, the efficiency of the above processes may become comparable. At high metallicity the decrease of PAH abundance is not compensated by VSG destruction, and the $F_{8} / F_{24}$ ratio goes down with age. These assumptions are preliminary and require further analysis, nevertheless this test allows to one to investigate evolutionary effects in PAH emission.

Acknowledgments. This work was supported by the RFBR grants 14-02-31456 and 14-0200604 and by the Russian Academy of Sciences program OFN-17. 

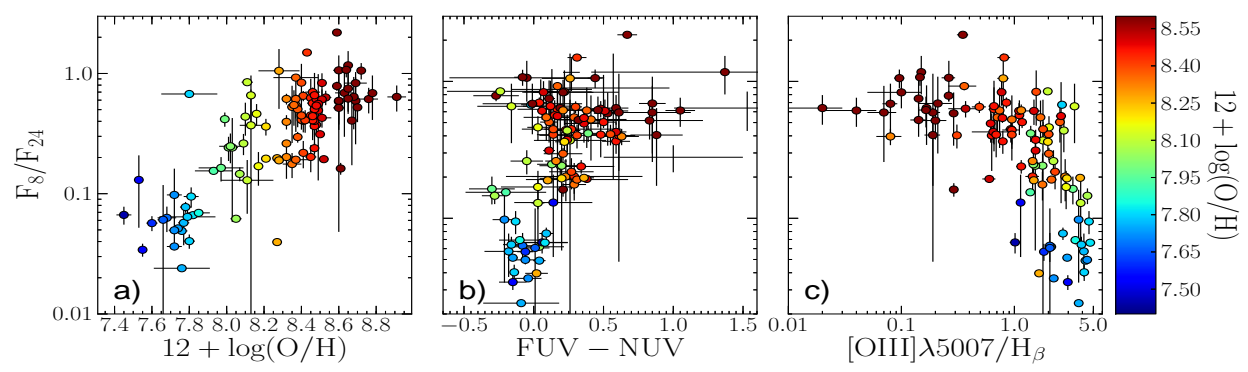

Figure 1: a) The $F_{8} / F_{24}$ ratio as a function of metallicity; b) and c): the $F_{8} / F_{24}$ ratio as a function of the radiation field hardness (FUV-NUV and $\left.I([O I I I] \lambda 5007) / I\left(H_{\beta}\right)\right)$.

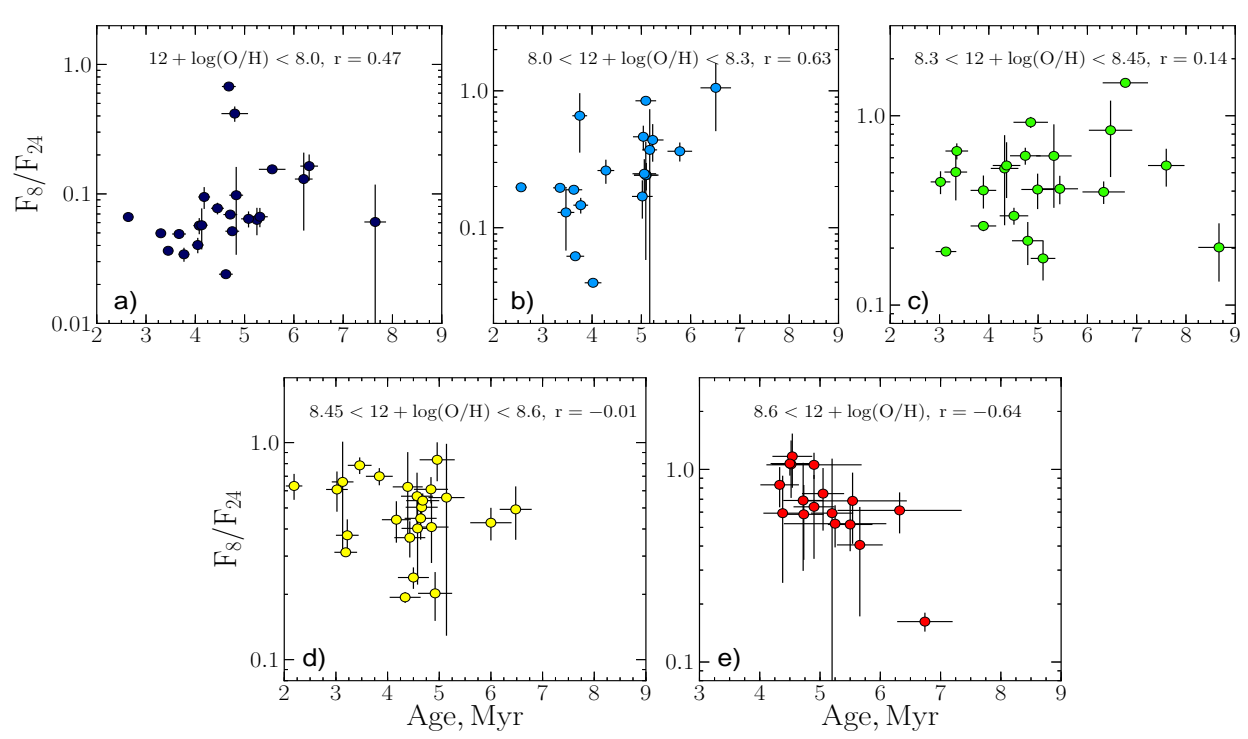

Figure 2: The $F_{8} / F_{24}$ ratio as a function of age.

\section{References}

[1] B. T. Draine et al., Dust Masses, PAH Abundances, and Starlight Intensities in the SINGS Galaxy Sample, ApJ (2007), 663, 866

[2] F. Galliano et al., Stellar Evolutionary Effects on the Abundances of Polycyclic Aromatic Hydrocarbons and Supernova-Condensed Dust in Galaxies, Apj, (2008), 672, 214

[3] M. S. Khramtsova et al. Polycyclic aromatic hydrocarbons in spatially resolved extragalactic star-forming complexes, MNRAS, (2013), 431, 2006

[4] E. M. Levesque et al. The Host Galaxies of Gamma-ray Bursts. II. A Mass-metallicity Relation for Long-duration Gamma-ray Burst Host Galaxies, AJ (2010) 140, 1557

[5] S. C. Madden, Effects of massive star formation on the ISM of dwarf galaxies, NewAR, (2000), 44, 249 